\title{
A MODELLING OF LINE TRANSFER IN SUPERGIANT ENVELOPES
}

\author{
Rrzysztof Gesicki \\ N. Copernicus Astronomical Center \\ Laboratory for Astrophysics \\ ul. Chopina $12 / 18$ \\ 87-100 Torun, Poland
}

\begin{abstract}
A computer code that solves the transfer equation in moving atmosphere is prepared for analysis of variability in circumstellar spectral lines. This model gives a theoretical line shape, which consists of a symmetric photospheric line and superposed on it a P-Cye type component originated in the envelope. Comparison between calculated and observed line profiles will be used to analyze $6 \mathrm{~A} / \mathrm{mm}$ spectra of $\mathrm{F}-\mathrm{K}$ supereiants.
\end{abstract}

\section{INTRODUCTION}

It is well known that some of the high luminous $F-K$ supergiants are surrounded by circumstellar envelopes. Circumstellar shells of Rho Cas and HR8752 were investigated by Sargent (1961), Smolinski and Climenhaga (1985), Lambert et al. (1981). One of indicators of circumstellar matter is the observed splitting in low-excitation-potential spectral lines. The interpretation of this feature is that a single and symmetric line formed in the photosphere changes its shape in the process of scattering while passing through the envelope. As the envelope is spherical, extended and expanding, the photospheric profile is affected by an absorption component shifted towards shorter wavelengths as well as by an emission component shifted towards longer wavelengths. The resulting profile is complicated and its analysis requires model computations. Such analysis was made for late type superilants by Sanner (1976), Bernat (1977) and by Hagen (1978).

\section{THE CHOSEN METHOD AND THE RESULTING CODE}

In my analysis of $6 \mathrm{~A} / \mathrm{mm}$ spectra of supergiants I have concentrated at first on Rho Cas (F8Ia+), which shows interesting changes in the shell line profiles (Fig.1). Havine decided to study the variability on the basis of a model computations I have written a computer code. There are many ways of handling line transfer in circumstellar shells, amone known authors are Minalas, Kunasz, Hummer, recently Sanner, Bertout. 


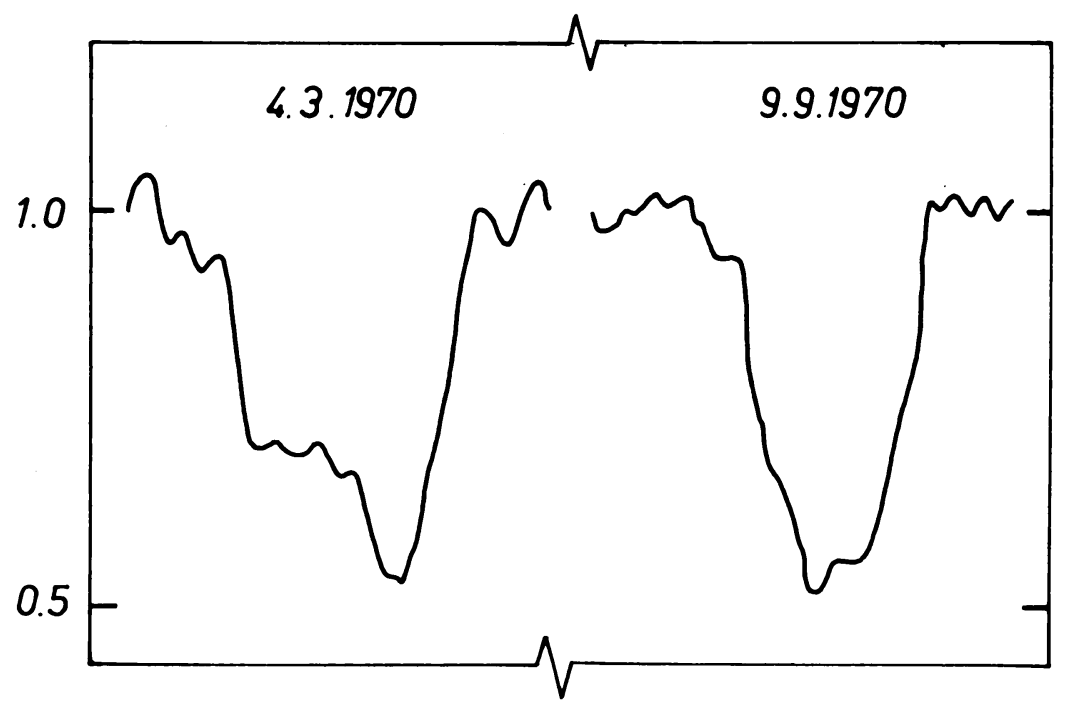

Figure 1. An example of 1 ine Ba II $5854 \mathrm{~A}$ in supergiant Rho Cas. The variability of the shortwave component, which is of circumstellar origin, is readily visible.
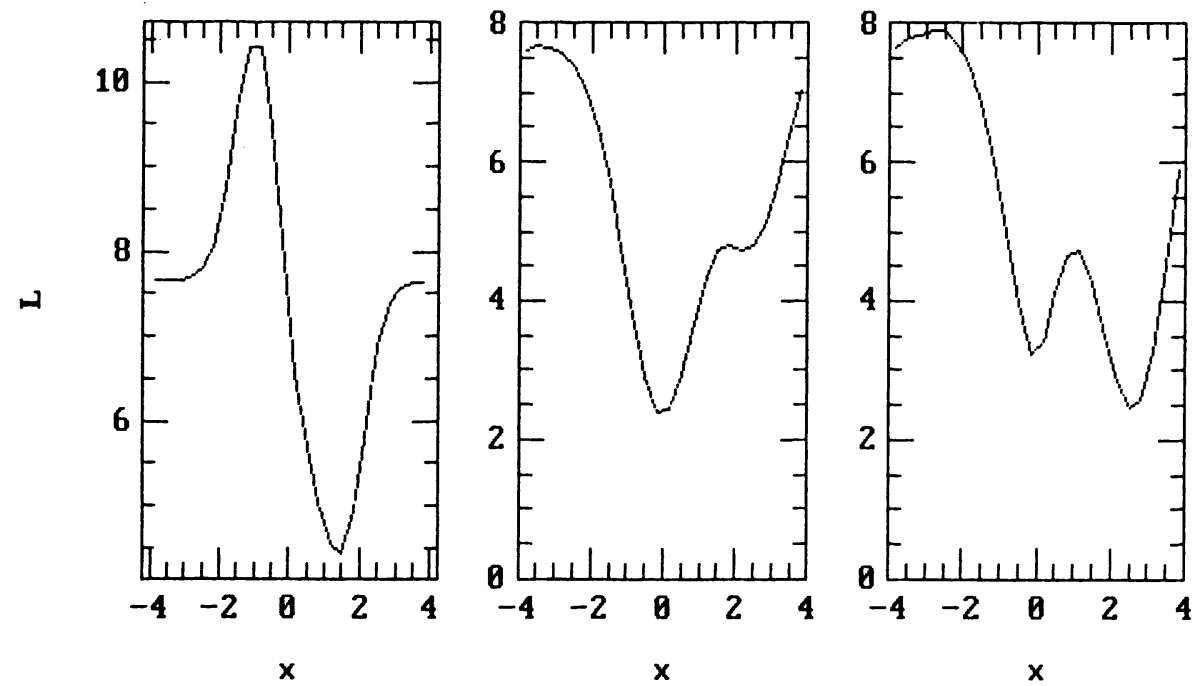

Figure 2. Luminosity profiles produced with the code. The horizontal axis is the frequency $x$ in Doppler units. First from left is the profile obtained with constant stellar continuum then follow two profiles with underlyine absorption line and with different mean opacity $T$ of the expanding envelope, $T=1$ and 3 respectively. 
I have chosen a method of Kunasz and fummer (1974) since it has already been successfully used to perform similar analysis (Bernat, Hacen).

The transfer equation is written in the observer's frame alone a set of rays parallel to the direction to the distant observer. Intersections of these rays with the set of radil wich divide the envelope into concentric shel1s, constitute the orid used to numerical solution. Spherical symmetry and the application of Feautrier variables result in an efficient code that does not require large computer memory. This method allows for an arbitrary density and velocity profile across the envelope. I have changed the boundary conditions so that one boundary is in the middle of the envelope. It enables to put the star surface inside the envelope. This version allows for an arbitrary shape of the underlying photospheric continuum which can be in the form of an absorption line.

The code is written in FORTRANTT and runs on an IBM PC/XT computer. some examples of line profiles obtained with it are shown in Fib.2. They represent the preliminary results. However a comparison between Fig.1 and Fis.2 shows that the model produces results similar to observations. The analysis of variability of supergiant envelopes requires

a construction of a whole atlas of theoretical profiles to compare with real spectra. This work is in procress.

\section{ACKADWLEDGDMITIS}

The computations were partially performed on an IEM XT compatible computer, on loan from Princeton University.

\section{RTFTRTICTS}

Bernat, A.P., Astrophys.J., 213, 756, (1977)

Hagen, W., Astrophys.J.Suppl., 38, 1, (1978)

Kunasz, P.B., Humer, D.G., Mon.Not.R.Astr.SoC., 166, 57, (1974)

Lambert, D.L., Hinkle, K.H., Hall D.K.B., Astrophys.J., 248, 638, (1981)

Sanner, F., Astrophys.J.Suppl., 32, 115, (1976)

Sargent, W.L.W., Astrophys.J., 134, 142, (1961)

Smolinsk1, J., Cl imenhaga, J.L., Proc. IAU Symp.No.116 (1985)

\section{DISCUSSION}

PRADERIE Is there an observational or theoretical evidence for a finite optical thickness $T$ in these circumstellar envelopes ?

GESICKI Presence of line splitting in low-excitation potential spectral lines and the absence of the splitting in other lines is one of the indicators of circumstellar shell. Calculations, such as presented by me, will allow to estimate the optical thickness. Earlier estimations based on the curve of growth method cannot be accurate. 